\title{
Diversity of Bee Flora in University of Agricultural Sciences, Dharwad Campus, Karnataka, India
}

\author{
K. C. Vidya ${ }^{1 *}$, Subhash B. Kandakoor ${ }^{2}$, S. T. Prabhu ${ }^{1}$ and S. C. Talekar ${ }^{3}$ \\ ${ }^{1}$ Department of Agricultural Entomology, College of Agriculture, Dharwad \\ Karnataka, India \\ ${ }^{2}$ Farm Superintendent and Head ARS, Bailhongal, India \\ ${ }^{3}$ AICRP on Rice, ARS, Mugad, India \\ *Corresponding author
}

\section{A B S T R A C T}

\begin{tabular}{|l|}
\hline Ke y w or d s \\
Diversity of Bee \\
Flora, UAS \\
Dharwad campus \\
\hline Article Info \\
$\begin{array}{l}\text { Accepted: } \\
\text { 24 October } 2020 \\
\text { Available Online: } \\
\text { 10 November } 2020\end{array}$ \\
\hline
\end{tabular}

\section{Introduction}

Pollination is a "free ecological service" which plays a vital role in the ecosystem by providing services to crops and wild plants. Pollination is a key part of global diversity. However in recent days, we found decline in both domesticated and wild pollinators due to environmental pollution, fragmentation of land, increased pest and disease, genetic diversity and variation in environmental conditions like rainfall, relative humidity and temperature. Pollinator decline impacting on two broad groups of flowering plants viz. crop

\begin{abstract}
Bees execute a special performance by pollinating several plant species. But the activity of honeybees depends on the bee flora availability and quantity and quality of nectar, pollen present in them. In the present study University of agricultural Sciences, Dharwad campus were surveyed to asses the diversity of bees' flora from July 2019- March 2020 which is covered with Agro- horticultural crops, wild plants and trees. Total 93 plants were considered as a bee flora, out of which 17 were field crops, 16 were horticultural crops, 33 were ornamental plants, 10 were trees and 17 were weeds. These bee flora available in different months supplying pollen, nectar and some both. Among the recorded bee flora, nectar and pollen yielding plants were more followed by nectar yielding and pollen yielding. It was also noticed that March month recorded with highest abundance of bee flora of 45.16 per cent recording 33 plant species.
\end{abstract}

plants and flowering plants. The plants which require obligate cross pollination by external agents, decline parallely with respective pollinator group (Potts et al., 2010). The goal of every organism on this earth including plants, is to reproduce and continue the generation. Plants continue their generation by producing seeds, for which flowers are the tools. Interestingly, many plants flower rely on the external agents to get pollinate; these external agents are called as vectors which helps in pollen movement from one flower to the other flower which results in fertilization followed by seed set. These vectors include 
water, wind, birds, insects and other animals which depends on the ecosystem. Among the animals, insects are the major pollinators providing the pollination services. These pollinators uphold the biodiversity and their population indicates healthy ecosytem.

\section{Materials and Methods}

During the survey period, in the UAS, Dharwad Campus, the flora was divided into groups consisting of field crops, horticultural crops, ornamental plants, weeds and forest trees. To determine the spectrum of different bee species visiting the blossoms of plants, pollination observations were made between 7 am to 12 noon during flowering period of the respective groups at every fortnight. During the visit, the flora was recorded that supported the bee population either as pollen source or nectar or both pollen and nectar.

For each pollination observation, ten minutes were spent at a particular flower plant and noted any bee that visited on it. After 10 minutes of observation, the bees were collected, killed and stored for identification. Initially the bees visitation data was examined qualitatively to determine whether the bees preferentially visit to particular flower types.

Classification of plant as nectariferous and polleniferous depends on activities performed by honeybees on different flowers. After landing on flower if honey bee sits calmly and extends its proboscis into the flower or collect the nectar from outside nectaries the flora was considered as nectariferous flora and if bees are hyperactive inside the flower carrying pollen on their body or in pollen basket (hind leg) from the flower, the flora were considered as polleniferous flora.

Flowers with honey bees showing both the activities were considered under nectariferous as well as polleniferous flora.
During the survey, the peak time of flowering, duration of flowering and the source of pollen or nectar was noted. Later the data was correlated to the diversity and activity of pollinator fauna and weather parameters. The survey was continued for entire study period at every fortnight on field crops, horticultural crops, ornamental plants, trees and weeds etc.

Finally, the recorded flora was collected and with help of plant taxonomist, they were identified. The total bee flora was categorised using available informations as a nectar yielders, pollen yielders and both pollen and nectar yielders. Then plants were identified monthwise in supporting as a food source through out the study period and all groups and their per cent contribution in each month was made for easy understanding like a floral calendar.

Per cent abundance of bee
flora for a month $=$

\section{Results and Discussion}

\section{Bee flora recorded at UAS, Dharwad Campus}

The documented flora showed the presence of 93 plant species belonging to different botanical families. Among the recorded families, Asteraceae consisted the highest number with 16 species, followed by Fabaceae with 14 species. These families includes field crops, ornamental crops, weeds, horticultural crops and perennial trees (Table 1).

\section{Availability of bee flora in different months}

Bee flora availability was recorded to asses the peak period and lean period of flora in the campus in different months of the year. This study will help to focus on the activity of the bees and to think about future prospectus of 
enhancing the bees diversity to support the better pollination process in field and horticultural crops. Based on the monthwise observations it was noticed that July and August months supported very less with very few floral availability compare to other months. In July, hardly 11 plants species were recorded as compared to total of 93 bee flora species Nevertheless the availability of flora increased from August and reached peak in the month of September and October with 40 and 37 plant species respectively. Futhermore second peak was observed in the month of March with 42 plant species (Table 2).

\section{Categorization of bee flora on reward basis}

The pollinators and plants are mutually benefitted from pollination process. Plants reward pollinators with pollen or nectar or both pollen and nectar and in turn plants get successful pollination service for better seed set. Among 93 plant species recorded in the UAS, Dharwad Campus, 54 plant species are with both pollen and nectar which belongs to the different groups (field crops, horticultural crops, trees and weeds) followed by 22 plant species which are nectar providers and 16 plant species are pollen providers. Garlic vine (Mansoa alliacea) neither pollen or nectar source on which Apis dorsata and Xylocopa amethystine were found (Table 3).

\section{Abundance of bee flora}

The existence of plant species with particularly attractive colours were important for the attraction of pollinators and the frequency of their visits. Richness of plant species i.e, cover of blossom and the existence of plant species which are attractive to increase the stability in the pollinator visitors frequency. The highest floral abundance was recorded during the month of March with 45.16 per cent followed by September with 43.01 per cent, October with
39.78 per cent. These three months were recorded as a highest floral abundance months which were directly related to the pollinators population and their visits. Similarly, January month recorded with 33.33 per cent, Months of November and February recorded the same per cent of floral abundance of 31.18 per cent and December 26.88 per cent and these four months were considered as a moderate level of floral abundance. August and July month recorded least floral abundance with 16.12 and 11.82 per cent respectively (Fig. 1).

\section{Abundance of field crops and horticultural crops contribution}

Out of 17, highest numbers were recorded in the month of September with 9 species. Followed by January and February with 6 species, October, November, December and March with 5 species, August with 3 species. But, July month with no flowering field crops were recorded during the study period. When, it was observed for total floral abundance of field crops with over all bee flora recorded, the September month was found as highest floral abundance contribution with 22.50 per cent followed by February with 20.68 per cent, December with 20 per cent, August with 20 per cent, January with 19.35 per cent, November with 17.24 per cent, October with 13.51 per cent, March with 11.90 per cent, July with zero abundance because of out of 11 flora recorded, none of them were field crops (Table 4 and 5).

During the study period a total of 16 horticultural crops were recorded, of which the highest numbers were recorded in the month of October with 7 horticultural crops. Followed by March with 6 species, September and January with 5 species, February with 4 species, July, August and December with 2 species, November with 1 species respectively. When, it was observed for total floral abundance of horticultural crops with 
over all bee flora, October month was found as highest floral abundance with 18.91 per cent followed by July with 18.18 per cent, January with 16.12 per cent, March with 14.28 per cent, February with 13.79 per cent,
August with 13.33 per cent, September with 12.50 per cent and December with 8.00 per cent, November found least abundance with 3.57 per cent respectively (Table 5).

Table.1 List of bee flora recorded at UAS, Dharwad Campus, July 2019 to March 2020

\begin{tabular}{|c|c|c|c|}
\hline Sl. No. & Scientific name & Common name & Family \\
\hline 1 & Cajanus cajan & Redgram & Fabaceae \\
\hline 2 & Cicer arietinum & Bengalgram & Fabaceae \\
\hline 3 & Vigna mungo & Blackgram & Fabaceae \\
\hline 4 & Vigna radiate & Greengram & Fabaceae \\
\hline 5 & Vigna unguiculata & Cowpea & Fabaceae \\
\hline 6 & Macrotyloma uniflorum & Horsegram & Fabaceae \\
\hline 7 & Glycine $\max$ & Soyabean & Fabaceae \\
\hline 8 & Albizia odoratissima & Black siris & Fabaceae \\
\hline 9 & Mimosa pudica & Touch me not & Fabaceae \\
\hline 10 & Caesalpinia & Peacock flower & Fabaceae \\
\hline 11 & Clitoria ternatea & Butterfly pea & Fabaceae \\
\hline 12 & Tamarindus indica & Tamarind & Fabaceae \\
\hline 13 & Phanera purpurea & Camel foot tree & Fabaceae \\
\hline 14 & Samanea saman & Rain tree & Fabaceae \\
\hline 15 & Helianthus annus & Sunflower & Asteraceae \\
\hline 16 & Carthamus tinctorius & Safflower & Asteraceae \\
\hline 17 & Guizotia abyssinica & Niger & Asteraceae \\
\hline 18 & Tridax procumbens & Tridax daisy & Asteraceae \\
\hline 19 & Parthenium hysterophorous & Santa Maria feverfew & Asteraceae \\
\hline 20 & Syndrella nodiflora & Cyndrella Weed & Asteraceae \\
\hline 21 & Zinnia & Zinnia & Asteraceae \\
\hline 22 & Tagetus erecta & Marigold & Asteraceae \\
\hline 23 & Callistephus chinensis & China aster & Asteraceae \\
\hline 24 & Cosmos & Cosmos & Asteraceae \\
\hline 25 & Gaillardia & Blanket flower & Asteraceae \\
\hline 26 & Symphyotrichum oblongifolium & Aromatic aster & Asteraceae \\
\hline 27 & Pulicaria vulgaris & False fleabane & Asteraceae \\
\hline 28 & Glebionis coronaria & Crown daisy & Asteraceae \\
\hline 29 & Ageratum houstonianum & Floss weed & Asteraceae \\
\hline 30 & Wedelia chinensis & Kalsarji & Asteraceae \\
\hline 31 & Lycopersicum esculentum & Tomato & Solanaceae \\
\hline 32 & Solanum melongena & Brinjal & Solanaceae \\
\hline 33 & Capsicum annum & Chilli & Solanaceae \\
\hline 34 & Nicandra physalodes & $\begin{array}{l}\text { Apple-of-Peru } \\
\text { Shoo-fly plant }\end{array}$ & Solanaceae \\
\hline
\end{tabular}




\begin{tabular}{|c|c|c|c|}
\hline 35 & Amaranthus retroflexus & Amaranth & Amaranthaceae \\
\hline 36 & Alternenthra sessilis & Sessile joyweed & Amaranthaceae \\
\hline 37 & Celosia argentea & Cock's comb & Amaranthaceae \\
\hline 38 & Achyranthes aspera & Devil's horsewhip & Amaranthaceae \\
\hline 39 & Convulvulus arvernsis & Field bindweed & Convolvulaceae \\
\hline 40 & Stictocardia beraviensis & Hawaiian Bells & Convolvulaceae \\
\hline 41 & Ipomea tricolor & Morning glory & Convolvulaceae \\
\hline 42 & Ipomoea horsfalliae & Cardinal creeper & Convolvulaceae \\
\hline 43 & Duranta erecta & Pigeon berry & Verbenaceae \\
\hline 44 & Lantana camera & Lantana & Verbenaceae \\
\hline 45 & Sorghum bicolour & Sorghum & Poaceae \\
\hline 46 & Zoysia spp & Zoysia & Poaceae \\
\hline 47 & Zea mays & Maize & Poaceae \\
\hline 48 & Fagopyrum esculentum & Buckwheat & Polygonaceae \\
\hline 49 & Antigon leptipus & Mexican creeper & Polygonaceae \\
\hline 50 & Gossypium & Cotton & Malvaceae \\
\hline 51 & Abelmoschus esculentus & Bhendi & Malvaceae \\
\hline 52 & Aleca rosea & Hollyhock & Malvaceae \\
\hline 53 & Allium сера & Onion & Amarylidaceae \\
\hline 54 & Zephyranthes sulphurea & Rain lily & Amarylidaceae \\
\hline 55 & Hymenocallis littoralis & Beach spider lilly & Amarylidaceae \\
\hline 56 & Cucumis sativus & Cucumber & Cucurbitaceae \\
\hline 57 & Coccinia grandis & Ivy guard & Cucurbitaceae \\
\hline 58 & Chrozophora rottleris & Suryavarti & Euphorbiaceae \\
\hline 59 & Euphorbia mili & Christ plant & Euphorbiaceae \\
\hline 60 & Asystasia gangetica & Chinese violet & Acanthaceae \\
\hline 61 & Thunbergia grandiflora & White sky vine & Acanthaceae \\
\hline 62 & Thunbergia erecta & Bush clock vine & Acanthaceae \\
\hline 63 & Tecoma stans & Yellow bells & Bignoniaceae \\
\hline 64 & Mansoa alliacea & Garlic vine & Bignoniaceae \\
\hline 65 & Pyrosteiga venusta & Orange trumpet vine & Bignoniaceae \\
\hline 66 & Anacardium occidentale & Cashew nut & Anacardiaceae \\
\hline 67 & Mangifera indica & Mango & Anacardiaceae \\
\hline 68 & Psidium guajava & Guava & Myrtaceae \\
\hline 69 & Callistemon citrinus & Bottle brush & Myrtaceae \\
\hline 70 & Sesamum indicum & Sesamum & Pedaliaceae \\
\hline 71 & Linum usitatissimum & Linseed & Linaceae \\
\hline 72 & Coriandrum sativum & Coriander & Apiaceae \\
\hline 73 & Punica granatum & Pomegranate & Puniaceae \\
\hline 74 & Muntingia calabura & Singapore cherry & Mutingiaceae \\
\hline 75 & Sapindus emarginatus & Soapnut tree & Sapindaceae \\
\hline 76 & Aegle marmelos & Stone apple & Rutaceae \\
\hline 77 & Terminalia catappa & Kadu Badami & Combretacea \\
\hline
\end{tabular}




\begin{tabular}{|l|l|l|l|}
\hline $\mathbf{7 8}$ & Calotropis & Milk weed & Apocynaceae \\
\hline $\mathbf{7 9}$ & Trianthema portulocastrum & Gaintpig weed & Aizoacea \\
\hline $\mathbf{8 0}$ & Portulaca quadrifida & Chicken weed & Portulacaceae \\
\hline $\mathbf{8 1}$ & Turnera subulata & Damiana & Passifloraceae \\
\hline $\mathbf{8 2}$ & Tristellateia australlis & $\begin{array}{l}\text { Shower of gold } \\
\text { climber }\end{array}$ & Malphigiacea \\
\hline $\mathbf{8 3}$ & Boughenvillea & Paper flower & Nyctaginaceae \\
\hline $\mathbf{8 4}$ & Polianthes tuberose & Tuberose & Asparagaceae \\
\hline $\mathbf{8 5}$ & Cuphea hyssopifolia & Mexican heather & Lythraceae \\
\hline $\mathbf{8 6}$ & Hamelia patens & Fire bush & Rubiaceae \\
\hline $\mathbf{8 7}$ & Impatiens balsamina & Balsam & Balsiminaceae \\
\hline $\mathbf{8 8}$ & Mentha arvensis & Wild mint & Lamiaceae \\
\hline $\mathbf{8 9}$ & Brassica & Mustard & Brassicacea \\
\hline $\mathbf{9 0}$ & Cocos nucifera & Coconut & Arecaceae \\
\hline $\mathbf{9 1}$ & Moringa oleifera & Drumstick & Moringaceae \\
\hline $\mathbf{9 2}$ & Phyllanthes acidus & Goosberry & Phyllanthaceae \\
\hline $\mathbf{9 3}$ & Azadirachta indica & Neem & Meliaceae \\
\hline
\end{tabular}

Table.2 Bee flora recorded in different months at UAS, Dharwad Campus, July 2019 to April 2020

\begin{tabular}{|c|c|c|c|c|c|c|c|c|c|c|}
\hline Common name & Scientific name & July & Aug & Sept & Oct & Nov & Dec & Jan & Feb & Mar \\
\hline \multicolumn{11}{|l|}{ Field Crops } \\
\hline Sorghum & Sorghum bicolour & -- & -- & $* *$ & -- & -- & $* *$ & $* *$ & -- & -- \\
\hline Maize & Zea mays & -- & -- & $* *$ & -- & -- & -- & -- & -- & $* *$ \\
\hline Buckwheat & Fagopyrum esculentum & -- & -- & -- & $* *$ & $* *$ & $* *$ & -- & -- & $* *$ \\
\hline Cotton & Gossypium & -- & -- & -- & $* *$ & $* *$ & & $* *$ & $* *$ & -- \\
\hline Redgram & Cajanus cajan & -- & -- & -- & $* *$ & $* *$ & $* *$ & $* *$ & $* *$ & -- \\
\hline Bengal gram & Cicer arietinum & -- & -- & -- & -- & -- & -- & $* *$ & $* *$ & -- \\
\hline Black gram & Vigna mungo & -- & $* *$ & $* *$ & $* *$ & $* *$ & -- & -- & -- & -- \\
\hline Green gram & Vigna radiate & -- & $* *$ & $* *$ & -- & -- & -- & -- & -- & $* *$ \\
\hline Cowpea & Vigna unguiculata & -- & -- & $* *$ & $* *$ & $* *$ & -- & -- & $* *$ & -- \\
\hline Horse gram & Macrotyloma uniflorum & -- & -- & -- & -- & -- & -- & $* *$ & -- & -- \\
\hline Mustard & Brassica nigra & -- & -- & -- & -- & -- & $* *$ & -- & -- & -- \\
\hline Sunflower & Helianthus annuиs & -- & -- & $* *$ & -- & -- & $* *$ & -- & $* *$ & -- \\
\hline Safflower & Carthamus tinctorius & -- & -- & -- & -- & -- & -- & -- & $* *$ & $* *$ \\
\hline Soyabean & Glycine $\max$ & -- & & $* *$ & -- & -- & -- & -- & -- & $* *$ \\
\hline Sesamum & Sesamum indicum & -- & $* *$ & $* *$ & -- & -- & -- & -- & -- & -- \\
\hline Linseed & Linum usitatissimum & -- & -- & -- & -- & -- & -- & $* *$ & -- & -- \\
\hline Niger & Guizotia abyssinica & -- & -- & $* *$ & -- & -- & -- & -- & -- & -- \\
\hline \multicolumn{11}{|l|}{ Horticultural crops } \\
\hline Onion & Allium сера & -- & -- & -- & -- & -- & -- & -- & -- & $* *$ \\
\hline Cucumber & Cucumis sativus & -- & $* *$ & $* *$ & $* *$ & -- & -- & -- & -- & -- \\
\hline
\end{tabular}




\begin{tabular}{|c|c|c|c|c|c|c|c|c|c|c|}
\hline Bhendi & Abelmoschus esculentus & -- & -- & -- & $* *$ & & -- & -- & -- & -- \\
\hline Tomato & $\begin{array}{l}\text { Lycopersicum } \\
\text { esculentum }\end{array}$ & -- & -- & -- & -- & -- & -- & $* *$ & -- & -- \\
\hline Brinjal & Solanum melongena & -- & -- & -- & $* *$ & -- & -- & -- & $* *$ & -- \\
\hline Chilli & Capsicum annum & -- & -- & -- & $* *$ & -- & -- & -- & -- & -- \\
\hline Ivy guard & Coccinia grandis & -- & -- & -- & -- & -- & -- & -- & -- & $* *$ \\
\hline Coriander & Coriandrum sativum & -- & -- & $* *$ & $* *$ & -- & -- & $* *$ & $* *$ & -- \\
\hline Amaranth & Amaranthus retroflexus & -- & -- & -- & -- & -- & -- & -- & -- & $* *$ \\
\hline Guava & Psidium guajava & $* *$ & -- & -- & -- & -- & -- & -- & -- & $* *$ \\
\hline Pomegrante & Punica granatum & -- & -- & $* *$ & $* *$ & -- & -- & -- & -- & -- \\
\hline Mango & Mangifera indica & -- & -- & -- & -- & -- & -- & $* *$ & $* *$ & -- \\
\hline Gooseberry & Phyllanthes acidus & -- & -- & -- & -- & -- & -- & -- & -- & $* *$ \\
\hline Coconut & Cocos nucifera & $* *$ & $* *$ & $* *$ & $* *$ & $* *$ & $* *$ & $* *$ & $* *$ & $* *$ \\
\hline Drumstick & Moringa oleifera & -- & -- & $* *$ & -- & -- & -- & -- & -- & -- \\
\hline Cashew & Anacardium occidentale & -- & -- & -- & -- & -- & -- & $* *$ & $* *$ & -- \\
\hline \multicolumn{11}{|l|}{ Trees } \\
\hline Singapore cherry & Muntingia calabura & $* *$ & $* *$ & $* *$ & -- & -- & -- & $* *$ & $* *$ & $* *$ \\
\hline Stone apple & Aegle marmelos & -- & -- & -- & -- & -- & -- & -- & -- & $* *$ \\
\hline Tamarind & Tamarindus indica & -- & -- & -- & -- & -- & $* *$ & $* *$ & -- & -- \\
\hline Indian almond & Terminalia catappa & -- & -- & -- & -- & -- & -- & -- & $* *$ & -- \\
\hline Soapnut tree & Sapindus emarginatus & -- & -- & & $* *$ & $* *$ & $* *$ & -- & -- & -- \\
\hline Yellow bells & Tacoma stans & -- & -- & $* *$ & $* *$ & $* *$ & $* *$ & $* *$ & $* *$ & $* *$ \\
\hline Black siris & Albizia odoratissima & -- & -- & -- & -- & -- & -- & $* *$ & -- & -- \\
\hline Camel foot tree & Phanera purpurea & -- & -- & $* *$ & $* *$ & $* *$ & -- & -- & -- & -- \\
\hline Rain tree & Samanea saman & -- & -- & $* *$ & $* *$ & $* *$ & -- & -- & $* *$ & $* *$ \\
\hline Neem & Azadirachta indica & -- & -- & -- & -- & -- & -- & -- & -- & $* *$ \\
\hline \multicolumn{11}{|l|}{ Ornamental crops } \\
\hline Damiana & Turnera subulata & -- & -- & $* *$ & $* *$ & $* *$ & -- & -- & -- & -- \\
\hline White sky vine & Thunbergia grandiflora & $* *$ & $* *$ & $* *$ & $* *$ & $* *$ & $* *$ & $* *$ & $* *$ & $* *$ \\
\hline Zinnia & Zinnia sp. & -- & -- & $* *$ & $* *$ & -- & -- & -- & -- & -- \\
\hline Mexican creeper & Antigon leptipus & -- & -- & $* *$ & $* *$ & $* *$ & $* *$ & -- & -- & -- \\
\hline Beach spider lilly & Hymenocallis littoralis & $* *$ & $* *$ & -- & -- & -- & -- & -- & -- & -- \\
\hline Marigold & Tagetus erecta & -- & -- & $* *$ & $* *$ & $* *$ & $* *$ & $* *$ & -- & -- \\
\hline Hollyhock & Aleca rosea & -- & -- & -- & $* *$ & -- & -- & -- & -- & -- \\
\hline Peacock flower & Caesalpinia & -- & -- & -- & $* *$ & $* *$ & $* *$ & $* *$ & -- & -- \\
\hline China aster & Callistephus chinensis & -- & -- & -- & -- & -- & $* *$ & $* *$ & -- & -- \\
\hline $\begin{array}{l}\text { Shower of gold } \\
\text { climber }\end{array}$ & Tristellateia australlis & -- & -- & -- & -- & -- & -- & $* *$ & $* *$ & $* *$ \\
\hline Cosmos & Cosmos & -- & -- & $* *$ & $* *$ & $* *$ & -- & $* *$ & $* *$ & $* *$ \\
\hline Christ plant & Euphorbia mili & -- & -- & $* *$ & $* *$ & $* *$ & -- & -- & $* *$ & $* *$ \\
\hline Paper flower & Boughen villea & -- & -- & $* *$ & -- & -- & -- & -- & -- & $* *$ \\
\hline Tube rose & Polianthes tuberose & -- & -- & -- & -- & -- & $* *$ & $* *$ & -- & -- \\
\hline Bush clock vine & Thunbergia erecta & -- & -- & $* *$ & -- & -- & -- & -- & -- & -- \\
\hline
\end{tabular}




\begin{tabular}{|c|c|c|c|c|c|c|c|c|c|c|}
\hline Garlic vine & Mansoa alliacea & -- & $* *$ & -- & $* *$ & $* *$ & -- & -- & -- & -- \\
\hline Butterfly pea & Clitoria ternatea & -- & -- & -- & -- & $* *$ & -- & -- & -- & -- \\
\hline $\begin{array}{l}\text { Apple-of-Peru } \\
\text { Shoofly plant }\end{array}$ & Nicandra physalodes & -- & -- & -- & $* *$ & -- & -- & -- & -- & -- \\
\hline Mexican heather & Cuphea hyssopifolia & $* *$ & $* *$ & $* *$ & $* *$ & $* *$ & $* *$ & $* *$ & $* *$ & $* *$ \\
\hline Morning glory & Ipomea tricolor & -- & -- & $* *$ & $* *$ & $* *$ & $* *$ & $* *$ & $* *$ & $* *$ \\
\hline Blanket flower & Gaillardia & -- & -- & -- & -- & -- & $* *$ & $* *$ & $* *$ & $* *$ \\
\hline Hawaiian Bells & Stictocardia beraviensis & -- & -- & -- & -- & -- & $* *$ & $* *$ & $* *$ & $* *$ \\
\hline Fire bush & Hamelia patens & -- & -- & -- & -- & $* *$ & -- & -- & -- & -- \\
\hline Bottle brush & Callistemon citrinus & -- & -- & -- & $* *$ & $* *$ & $* *$ & -- & -- & -- \\
\hline Rain lily & Zephyranthes sulphurea & $* *$ & $* *$ & -- & -- & -- & -- & -- & -- & -- \\
\hline Aromatic aster & $\begin{array}{l}\text { Symphyotrichum } \\
\text { oblongifolium }\end{array}$ & -- & -- & -- & -- & -- & -- & -- & -- & $* *$ \\
\hline Pigeon berry & Duranta erecta & -- & $* *$ & $* *$ & -- & -- & -- & -- & $* *$ & $* *$ \\
\hline Cardinal creeper & Ipomoea horsfalliae & -- & -- & $* *$ & $* *$ & $* *$ & $* *$ & $* *$ & $* *$ & -- \\
\hline $\begin{array}{l}\text { Orange trumpet } \\
\text { vine }\end{array}$ & Pyrosteiga venusta & -- & -- & -- & -- & -- & -- & $* *$ & $* *$ & $* *$ \\
\hline Crown daisy & Glebionis coronaria & -- & -- & $* *$ & -- & -- & -- & -- & $* *$ & $* *$ \\
\hline Cockscomb & Celosia argentea & -- & -- & $* *$ & $* *$ & & -- & -- & -- & $* *$ \\
\hline False fleabane & Pulicaria vulgaris & -- & -- & -- & -- & -- & -- & $* *$ & -- & -- \\
\hline Balsam & Impatiens balsamina & -- & -- & $* *$ & -- & -- & -- & -- & -- & -- \\
\hline \multicolumn{11}{|l|}{ Weeds } \\
\hline Milk weed & Calotropis & -- & -- & -- & -- & -- & -- & -- & $* *$ & $* *$ \\
\hline Chinese violet & Asystasia gangetica & -- & -- & -- & -- & -- & $* *$ & $* *$ & -- & -- \\
\hline Field bind weed & Convulvulus arvernsis & -- & -- & -- & -- & -- & -- & -- & $* *$ & $* *$ \\
\hline Suryavarti & Chrozophora rottleris & -- & -- & -- & -- & -- & -- & -- & -- & $* *$ \\
\hline Kalsarji & Wedelia chinensis & $* *$ & $* *$ & $* *$ & $* *$ & $* *$ & -- & -- & -- & -- \\
\hline Touch me not & Mimosa pudica & -- & -- & $* *$ & -- & $* *$ & $* *$ & -- & -- & $* *$ \\
\hline Sessile joyweed & Alternenthra sessilis & -- & -- & $* *$ & $* *$ & $* *$ & $* *$ & -- & -- & $* *$ \\
\hline Lantana & Lantana camera & $* *$ & $* *$ & $* *$ & $* *$ & $* *$ & -- & -- & $* *$ & -- \\
\hline Tridax daisy & Tridax procumbens & $* *$ & -- & -- & $* *$ & $* *$ & -- & $* *$ & -- & $* *$ \\
\hline Wild mint & Mentha arvensis & -- & -- & $* *$ & -- & -- & -- & -- & -- & -- \\
\hline Zoysia & Zoysia sp. & -- & -- & -- & -- & -- & -- & -- & -- & $* *$ \\
\hline Gaint pigweed & $\begin{array}{l}\text { Trianthema } \\
\text { portulocastrum }\end{array}$ & $* *$ & $* *$ & -- & -- & -- & -- & -- & -- & $* *$ \\
\hline Cyndrella Weed & Syndrella nodiflora & -- & -- & -- & $* *$ & -- & -- & -- & -- & $* *$ \\
\hline Devil's horsewhip & Achyranthes aspera & -- & -- & -- & $* *$ & -- & -- & -- & -- & -- \\
\hline Chicken weed & Portulaca quadrifida & -- & -- & -- & -- & -- & -- & -- & -- & $* *$ \\
\hline Floss weed & Ageratum houstonianum & -- & -- & -- & -- & -- & -- & -- & -- & $* *$ \\
\hline $\begin{array}{l}\text { Santa Maria } \\
\text { feverfew }\end{array}$ & $\begin{array}{l}\text { Parthenium } \\
\text { Hysterophorous }\end{array}$ & -- & -- & $* *$ & -- & -- & -- & -- & -- & $* *$ \\
\hline
\end{tabular}


Table.3 Bee flora and their reward in UAS, Dharwad Campus, July 2019 to March 2020

\begin{tabular}{|c|l|l|l|}
\hline Sl. No. & Pollen yielding plants & Nectar yielding plants & \multicolumn{1}{|c|}{ Pollen and nectar yielding plants } \\
\hline $\mathbf{1}$ & Sorghum bicolor & Fagopyrum esculentum & Gossypium \\
\hline $\mathbf{2}$ & Zea mays & Cajanus cajan & Macrotyloma uniflorum \\
\hline $\mathbf{3}$ & Lycopersicum esculentum & Cicer arietinum & Brassica \\
\hline $\mathbf{4}$ & Solanum melongena & Vigna mungo & Helianthus annus \\
\hline $\mathbf{5}$ & Psidium guajava & Vigna radiate & Carthamus tinctorius \\
\hline $\mathbf{6}$ & Zinnia sp. & Vigna unguiculata & Sesamum indicum \\
\hline $\mathbf{7}$ & Antigon leptipus & Glycine max & Linum usitatissimum \\
\hline $\mathbf{8}$ & Aleca rosea & Phanera purpurea & Guizotia abyssinica \\
\hline $\mathbf{9}$ & Nicandra physalodes & Turnera subulata & Allium cepa \\
\hline $\mathbf{1 0}$ & Ipomea tricolor & Thunbergia grandiflora & Cucumis sativus \\
\hline $\mathbf{1 1}$ & Convulvulus arvernsis & Hymenocallis littoralis & Abelmoschus esculentus \\
\hline $\mathbf{1 2}$ & Mimosa pudica & Tagetus erecta & Capsicum annum \\
\hline $\mathbf{1 3}$ & Achyranthes aspera & Stictocardia beraviensis & Coccinia gramdis \\
\hline $\mathbf{1 4}$ & Portulaca quadrifida & Zephyranthes sulphurea & Coriandrum sativum \\
\hline $\mathbf{1 5}$ & Mangifera indica & Ipomoea horsfalliae & Amaranthus retroflexus \\
\hline $\mathbf{1 6}$ & Samanea saman & Pyrosteiga venusta & Punica granatum \\
\hline $\mathbf{1 7}$ & & Chrozophora rottleris & Tamarindus indica \\
\hline $\mathbf{1 8}$ & & Alternenthra sessilis & Muntingia calabura \\
\hline $\mathbf{1 9}$ & & Lantana camera & Aegle marmelos \\
\hline $\mathbf{2 0}$ & & Mentha arvensis & Terminalia catappa \\
\hline & & & \\
\hline & & &
\end{tabular}

Table.4 Bee flora contribution (Abundance) in field crops and horticultural crops, July 2019 to March 2020

\begin{tabular}{|l|c|c|c|c|c|}
\hline \multicolumn{1}{|c|}{ Month } & \multicolumn{2}{|c|}{ Bee flora } & Total bee flora & \multicolumn{2}{c|}{ Floral Abundance of field crops (\%) } \\
\cline { 1 - 3 } & FC & HC & & FC & HC \\
\hline July & 0 & 2 & 11 & 0.00 & 18.18 \\
\hline Aug & 3 & 2 & 15 & 20.00 & 13.33 \\
\hline Sept & 9 & 5 & 40 & 22.50 & 12.50 \\
\hline Oct & 5 & 7 & 37 & 13.51 & 18.91 \\
\hline Nov & 5 & 1 & 29 & 17.24 & 3.44 \\
\hline Dec & 5 & 2 & 25 & 20.00 & 8.00 \\
\hline Jan & 6 & 5 & 31 & 19.35 & 16.12 \\
\hline Feb & 6 & 4 & 29 & 20.68 & 13.79 \\
\hline Mar & 5 & 6 & 42 & 11.90 & 14.28 \\
\hline Overall & $\mathbf{1 7}$ & $\mathbf{1 6}$ & $\mathbf{9 3}$ & $\mathbf{1 8 . 2 7}$ & $\mathbf{1 7 . 2 0}$ \\
\hline
\end{tabular}


Table.5 Bee flora contribution (Abundance) in ornamental plants, trees and weeds, July 2019 to March 2020

\begin{tabular}{|c|c|c|c|c|c|c|c|}
\hline Month & \multicolumn{3}{|c|}{ Bee flora } & \multicolumn{2}{c|}{$\begin{array}{c}\text { Total bee } \\
\text { flora }\end{array}$} & \multicolumn{3}{|c|}{ Floral Abundance (\%) } \\
\hline & OP & T & W & OP & T & W \\
\hline July & 4 & 1 & 4 & 11 & 36.36 & 9.09 & 36.36 \\
\hline Aug & 6 & 1 & 3 & 15 & 40.00 & 6.66 & 20.00 \\
\hline Sept & 16 & 4 & 6 & 40 & 40.00 & 10.00 & 15.00 \\
\hline Oct & 16 & 4 & 5 & 37 & 43.24 & 10.81 & 13.51 \\
\hline Nov & 14 & 4 & 5 & 29 & 48.27 & 13.79 & 17.24 \\
\hline Dec & 12 & 3 & 3 & 25 & 48.00 & 12.00 & 12.00 \\
\hline Jan & 14 & 4 & 2 & 31 & 45.16 & 12.90 & 6.45 \\
\hline Feb & 12 & 4 & 3 & 29 & 41.37 & 13.79 & 10.34 \\
\hline Mar & 14 & 4 & 12 & 42 & 33.33 & 9.52 & 28.57 \\
\hline Overall & $\mathbf{3 3}$ & $\mathbf{1 0}$ & $\mathbf{1 7}$ & $\mathbf{9 3}$ & $\mathbf{3 5 . 4 8}$ & $\mathbf{1 0 . 7 5}$ & $\mathbf{1 8 . 2 7}$ \\
\hline
\end{tabular}

Abundance of total bee flora recorded from July 2019 to March 2020

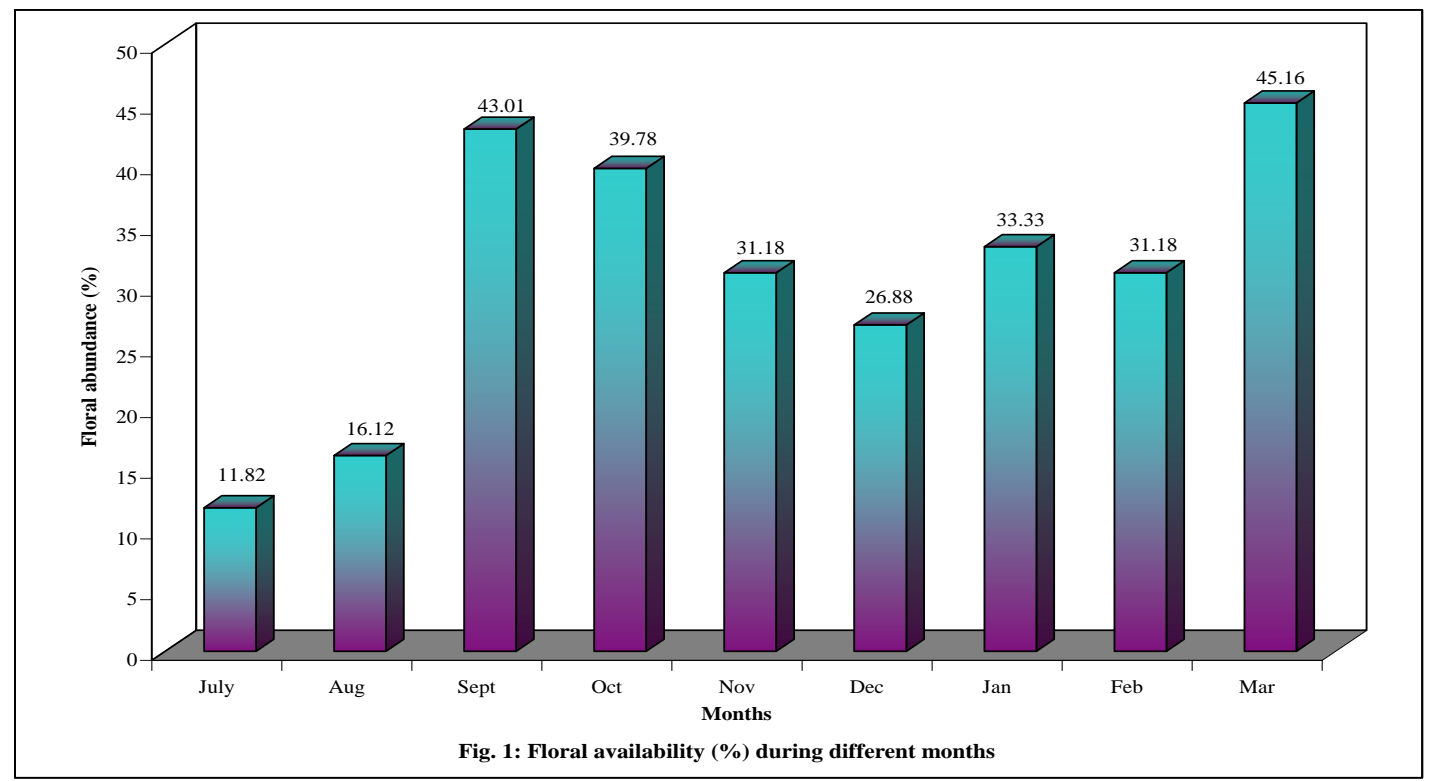

\section{Abundance of ornamental plants, trees and weeds contribution}

During the study period, a total of 33 ornamental plants were recorded as a bee flora. Out of 33, highest numbers were recorded in the month of September and October with 16 ornamental plants. Followed by November, January and March each with 14 species, December and February with 12 species, August with 6 species and July with 4 species. When, it was observed for total floral abundance of ornamental plants with over all flora, the November month were to be found highest floral abundance with 48.27 per cent followed by December with 48 per cent, January with 45.16 per cent, October with 43.24 per cent, February with 41.37, September and August each with 40 per cent, July with 36.36 per cent, March month 
reported least floral abundance of ornamental plants of 33.33 per cent (Table 6).

In total, during the study period, 10 trees were recorded as a bee flora. Out of 10, highest numbers were recorded in the month of September, October, November, January, February and March, all with 4 tree species, Followed by December with 3 tree species, July and August were recorded with only one tree species each. When it was observed for total floral abundance of trees with over all bee flora recorded, the November and February month found to be highest floral abundance each with 13.79 per cent followed by January with 12.90 per cent, December with 12 per cent, October with 10.81 per cent, September with 10 per cent, March with 9.52 per cent, July with 9.09 per cent, least floral abundance of trees reported during August month with only 6.66 per cent (Table 6).

In total, during the study period, 17 weeds were recorded as a bee flora. Out of 17, highest numbers were found in the month of March with 12 weeds. Followed by September with 6 weed species, October and November with 5 weed species, July with 4 weed species, August, December and February each with 3 weed species, January with 2 weed species. When, it was observed for total floral abundance of weeds with over all bee flora recorded, the highest abundance was noticed with 36.36 per cent observed in July, followed by March with 28.57 per cent, August with 20 per cent, November with 17.24 per cent, September with 15 per cent and January found least abundance with 6.45 per cent (Table 6).

Among the total bee flora which were catagorised into field crops, horticultural crops, ornamental plants, trees and weeds and out of these 93 plant species recorded, the ornamental plants contributed highest per cent of 35.48 floral abundance, followed by field crops and weeds with 18.27 per cent, followed by horticultural crops with 17.20 per cent and least in case of tree species which contributed only 10.75 per cent (Table 5).

As per the data, totally 93 plant species belonging to different botanical families were recorded, this study was compararble with the study of Pande and Ramkrushna (2018) in Nagpur and Wardha districts of Maharashtra where they recorded 92 plant species belongs to different families. When bee flora was observed month wise, it clearly showed that July and August supports very less with very few floral availability; March, September, October encouraged with high floral availability compare to other months, similar investigation done by Shiva et al. (2018), who also reported the maximum bee flora during March month (45) followed by September, February, April with 39 species, August with 36 species, May with 34 species, July with 32 species, January with 31 species, December with 30 species, October with 28 species, June with 27 species and November recorded with least number of bee flora with 25 species. Among the recorded 93 bee floral species, 54 plant species were both pollen and nectar yielders which belongs to the different groups (field crops, horticultural crops, trees and weeds) followed by 22 plant species which are nectar providers and 16 plant species are pollen providers. Bhalchandra et al., 2014 grouped identified flora into nectar, pollen and combination of both pollen and nectar. Total bee flora which were catagorised into field crops, horticultural crops, ornamental plants, trees, weeds and out of these 93 plant species recorded, the ornamental plants contributed highest per cent of 35.48 floral abundance with 33 bee flora. Followed by, field crops and Weeds with 18.27 per cent with 17 bee flora each, followed by horticultural crops with 17.20 per cent with 16 plants and least in case of tree species which contributed only 10.75 per cent 
with 10 species. Pande and Ramkrushna (2018) reported 69.4 per cent contribution from the cultivated plant species during August, 66.6 per cent in September, 60.7 per cent in October, 56.2 per cent in July, 51.6 per cent in January, 51.2 per cent in February, 51.1 per cent in March, 48.0 per cent in November, 46.6 per cent in December, 41.0 per cent in April and found lowest during May with $35.2 \%$. related to wild plants, it found reverse to the abundance of cultivated plants viz, highest during may month with 64.8 per cent and lowest during August with 30.6 per cent.

In conclusion the bees are necessarily important component in cropping system by pollinating wide variety of crops. This investigation represents 93 bee flora out of which 17 were field crops; 16 were horticultural crops; 33 were ornamental plants; 10 were trees and 17 were weeds. These groups were the provider of pollen, nectar and both pollen and nectar. Contribution from the source of Ornamental plants recorded highest with 35.48 per cent, field crops and weeds equally contribute to the bee source with 18.27 per cent, horticultural crops with 17.20 per cent and trees with 10.75 per cent recorded with least contribution. It can be concluded that UAS, Dharwad, Campus can be considered as rich bee floral area, where scientific bee keeping should be undertaken to improve the farmers livelihood. The record of wild bee flora during the study encourages their conservation for sustainable bee keeping in future as well.

\section{References}

Bhalchandra, W., Baviskar, R. K. and Nikam, T. B., 2014. Diversity of nectariferous and polleniferous bee flora at Anjaneri and Dugarwadi hills of Western Ghats of Nasik district (MS) India. $J$. Entomol. Zool. Stud., 2(4): 244-249.

Pande, R. and Ramkrushna, G. I., 2018, Diversification of Honey bees' flora and bee flora calendar for Nagpur and Wardha districts of Maharashtra, India. J. Entomol. Zool. Stud, 6(2): 3102-3110.

Potts, S. G., Biesmeijer, J. C., Kremen, C., Neumann, P., Schweiger, O. and Kunin, W. E., 2010, Global pollinator declines: trends, impacts and drivers. Trends Ecol. Evol, 25(6): 345353.

Shiva, P. Shiva, Resam, B. Thapa, Moha, D. Sharma, Shrawan, K. Sah and Yubak Dhoj, G. C., 2018, Bee floral calendar of cultivated and wild plants available in different agroecosystems of Chitwan, Nepal, Int. J. Res. Granthaalayah, 6(11): 222-245.

\section{How to cite this article:}

Vidya, K. C., Subhash B. Kandakoor, S. T. Prabhu and Talekar, S. C. 2020. Diversity of Bee Flora in University of Agricultural Sciences, Dharwad Campus Karnataka, India. Int.J.Curr.Microbiol.App.Sci. 9(11): 3365-3376. doi: https://doi.org/10.20546/ijcmas.2020.911.402 